\title{
Brane Solutions in Supergravity and the Near-Horizon Geometries
}

\author{
AdÉmọ́lÁ AdÉìfÉọBA \\ ITP, Universität Heidelberg
}

\begin{abstract}
This note represents a summary of a talk given at the 2018 Summer Mathematical Physics seminar, with focus on the physical and the mathematical concepts of the holography principle and large- $\mathrm{N}$ dualities. Here, we present an elementary overview of the $p$-brane solutions in supergravity, setting up a stage for a fairly broad topics on the AdS/CFT correspondence.
\end{abstract}

2018 Mathematical Physics Seminar: Uni-Heidelberg/Walcher/AdSCFT

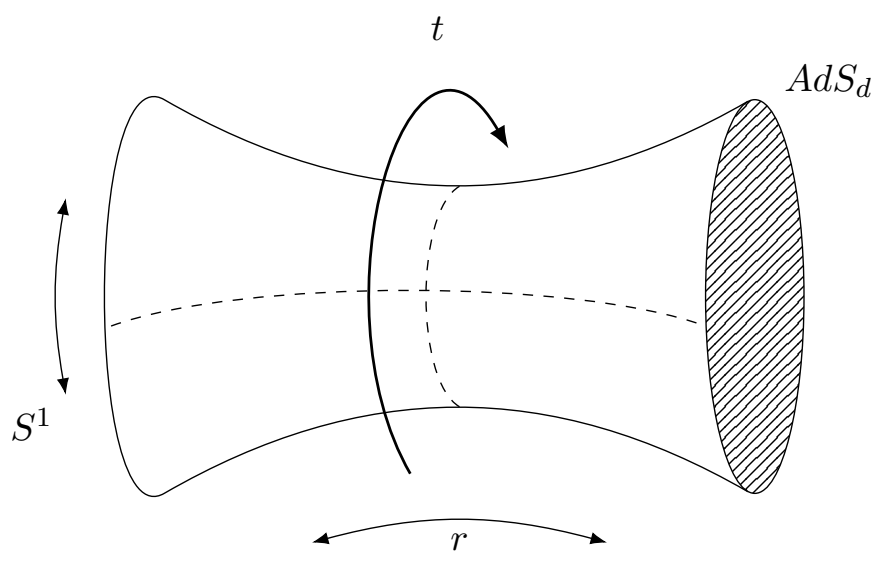




\section{Contents}

0 Introduction $\quad 2$

1 Black Holes: Towards $p$-branes $\quad 3$

1.1 Black Holes within GR . . . . . . . . . . . . . . . . . . . . . 3

1.1.1 Extremal RN Black Holes . . . . . . . . . . . . . . . . . . . . 5

1.1.2 Spacetime with a cosmological constant . . . . . . . . . . . . . 5

1.2 Extended Objects: $p$-Branes . . . . . . . . . . . . . . . . . 6

2 Elements of Supergravity $\quad 8$

2.1 Supergravity: Reminder . . . . . . . . . . . . . . . . . . . 8

2.2 Superstring/M-theory: Towards 10D and 11D SUGRA . . . . . . . . . . . 13

2.2.1 Massless Sectors of Superstring Theories . . . . . . . . . . . . . . . . 13

2.2.2 Massless Sectors of M-theory . . . . . . . . . . . . . . . . 15

3 The $p$-Brane Solution $\quad 15$

3.1 Extremal Supermembranes . . . . . . . . . . . . . . . . . . 20

3.2 Extremal Dp-branes . . . . . . . . . . . . . . . . . . . . 20

3.3 The Near-Horizon Geometries . . . . . . . . . . . . . . . . . . . . 22

3.4 Special Holonomy Manifold in M-theory . . . . . . . . . . . . . . . . . 23

\section{Introduction}

At the level of general relativity (GR), various spacetime configurations arise as generic solution to the Einstein field equations, some of which are known to be physical and thus play important roles in the discussion of physical problems. Examples include black hole configurations such as the Schwarzschild solution [1], the Reissner-Nordström metric [2], Kerr-Newman solution [3, 4], as well as the Friedmann-Lemaître-Robertson-Walker metric of the standard model of Big Bang cosmology. The inclusion of the cosmological constant led to some spacetime solutions that are asymptotically (A)dS. Example of such solution is the Kottler black holes [5], also known as the Schwarzschild-(A)dS solution. The failure of GR to adequately describe gravity, for instance, in some spacetime regime such as black hole singularity, led to the quest for a consistent theory of quantum gravity [6], and Supergravity (SUGRA) arise along the history of development with its important role in string theory. 
There are various reasons for studying the classical configuration in supergravity, and most especially, in higher dimensions. One is the possibility it offers for studying the non-perturbative structure of string theories through solitonic objects such as D-branes. D-branes are nothing but the extended objects in string theories on which open strings ends, and they have been identified with the classical solutions of the 10D SUGRA which are essentially the low energy limit of superstring theories. Alongside with the open strings that ends on it, the gravitational cloud of D-branes serves to provide a link between string theories and black hole physics.

Another motivation for studying $p$-brane solutions to higher dimensional supergravity theories is due to the gauge/gravity duality which provides new important tools for studying quantum field theories. This is at the heart of the discovery of the AdS/CFT correspondence [7, 8] which conjectures that string/M-theory on a certain SUGRA background geometry is dual to a conformal field theory on the boundary of the geometry. This remarkable discovery has been a subject of study for about two decades, and it has shared many suprising links with different areas of theoretical physics and mathematics. This is in fact the subject of our focus in this semester's mathematical physics seminar. Towards this ends, we shall review some important classical background solution of the various superstring/M-theories which are crucial in the study of the correspondence.

We begin in chapter 1 by introducing black holes and their extension into $p$-branes. We then provide some elements of supergravity in chapter 2 and highlight the low energy limit of superstrings and M- theories. The bosonic sector of this theory is what we eventually solved in chapter 3. The near horizon geometry of the elementary $p$-brane configurations are then introduced. Some brane configurations open up a path towards making contact with physics in lower dimension from superstring/M-theory via compactification. These are manifolds with special holonomies which retain some amount of supersymmetry. These configurations exhibit some interesting properties, such as mirror symmetry, that make them of special interest to both mathematics and physics. We shall briefly highlight a little of this towards the end.

\section{Black Holes: Towards $p$-branes}

Black holes arise as physical prediction of Einstein's GR. In four spacetime dimensions, they are just point-like, and in the absence of angular momentum have $\mathrm{SO}(1,3)$ symmetry. The boundary of this compact object, beyond which no causal signal can escape to infinity, is called the event horizon and it is crucial in a formal definition of black hole. We begin here by presenting some elementary class of classical black hole solutions with GR, and expand the concept to that of extended configurations in higher dimensions called $p$-branes.

\subsection{Black Holes within GR}

The simplest stationary solution to the vacuum Einstein-Maxwell equations (EFE) describing compact objects are spherically symmetric, having a metric of the form

$$
d s^{2}=-A(r) d t^{2}+\frac{d r^{2}}{B(r)}+r^{2} d \Omega_{2}^{2}
$$

where $d \Omega_{2}^{2}$ is the metric of the 2 -sphere, and $A(r)$ and $B(r)$ are some lapse functions. The first encounter with black hole solution was by Karl Schwarzschild who constructed a vacuum solution 
to the EFE. The geometry of this solution is Ricci flat, $R_{\mu \nu}=0$. Following Birkhoff's theorem, for $d \geq 4$, any $d$-dimensional spherically symmetric solution of the vacuum EFE belongs to the family of Schwarzschild metric parametrized by mass $M$. The Schwarzschild-Tangherlini solution is given as $[1,9]$

$$
d s^{2}=-\left(1-\frac{\mu}{r^{d-3}}\right) d t^{2}+\frac{d r^{2}}{1-\frac{\mu}{r^{d-3}}}+r^{2} d \Omega_{d-2}^{2},
$$

where

$$
\mu \equiv \frac{16 \pi G_{d} M}{(d-2) \Omega_{d-2}}
$$

and $G_{d}$ is the Newton's coupling in $d$-dimensions. In the limit $r \rightarrow \infty$, this solution approaches a Minkowski space, showing that it is asymptotically flat in the weak field limit. In this limit, one can recover the Newtonian gravity. The structure of this solution is well known. Apart from the existence of the event horizon, one can establish the existence of a curvature singularity by observing that in four dimensions, the Kretschmann invariant, $R_{\mu \nu \rho \sigma} R^{\mu \nu \rho \sigma}=48 G^{2} M^{2} / r^{6}$, diverges as $r \rightarrow 0$. At singularity, the regularity of the spacetime metric is lost and the energy density of the collapsing matter blows up. In essence, the classical description of gravity can no more provide an adequate description of spacetime in the neigbourhood of the singularity.

In four spacetime dimensions, the no hair theorem postulates that all black-hole solutions to the Einstein-Maxwell equations are uniquely characterized by just three observable classical parameters: the mass $(M)$, the electric charge $(Q)$, and angular momentum $(J)$, so that other black-hole solutions within GR are classifed in what follows.

- Reissner-Nordström (RN) or Charged Black Hole is the solution to EFE-Maxwell field equations for a spherically symmetric system with a radial electric field and zero 4-current density [2]. The metric takes the form

$$
f(r)=d s^{2}=-\frac{\left(r-r_{+}\right)\left(r-r_{-}\right)}{r^{2}} d t^{2}+\frac{r^{2}}{\left(r-r_{+}\right)\left(r-r_{-}\right)}+r^{2} d \Omega^{2},
$$

with event horizons located at the coordinate singularites

$$
r_{ \pm}=G M \pm \sqrt{G^{2} M^{2}-G Q^{2}} \text { for } M<|Q|(\text { Sub-Extremal }) .
$$

We note that for $M<|Q|$, the spacetime possesses a naked singularity at $r=0$. This is the super-Extremal limit. At extremality, where $M=Q$, the event horizons coincide at the extremal radius $r_{ \pm}=G M=|Q|$. In particular, the extremal black hole metric is given by

$$
d s^{2}=-\left(1-\frac{G M}{r}\right)^{2} d t^{2}+\left(1-\frac{G M}{r}\right)^{-2} d r^{2}+r^{2} d \Omega^{2}
$$

- Kerr Solution or Rotating Black Hole: represents the spinning generalization of the Schwarzschild solution and is relevant for astrophysical black holes [3]. The metric takes the the form

$$
d s^{2}=-\frac{\Delta}{\Xi}\left[d t-a \sin ^{2} \theta d \varphi\right]+\frac{\Xi}{\Delta} d r^{2}+\Xi d \theta^{2}+\frac{\sin ^{2} \theta}{\Xi}\left[\left(r^{2}+a^{2}\right) d \varphi-a d t\right]^{2}
$$

where

$$
\Delta:=r^{2}-2 G M r+a^{2}, \quad \Xi:=r^{2}+a^{2} \cos ^{2} \theta .
$$

This solution describes a rotating black hole with angular momentum $J=a M$. One can observe that in the limit of very large mass, the $a \rightarrow 0$, so that the Schwarzschild solution is recovered. 
- Kerr-Newman Solution: represents the spinning generalization of RN and the electrically charged Kerr solution [4]. The relevant metric is obtained by replacing $\Delta$ in the Kerr solution by

$$
\Delta_{Q}:=r^{2}-2 G M r+a^{2}+G Q^{2} .
$$

\subsubsection{Extremal RN Black Holes}

We have seen that the extremal limit of the RN black hole take the form (6). The maximal charge of this black hole is given by its mass. We shall later see that such solution for which $M=Q$ is realized are stable supersymmetric objects.

As a warm up, let us look at the near horizon limit of the extremal limit of the RN black hole solution. Define $\rho=r-G M$, solution (6) can be written as

$$
d s^{2}=-\left(1+\frac{r_{0}}{\rho}\right)^{-2} d t^{2}+\left(1+\frac{r_{0}}{\rho}\right)^{2}\left(d \rho^{2}+r_{0}^{2} d \Omega^{2}\right),
$$

where $r_{0}=G M$. In this way, the horizon of the extremal RN black hole is at $\rho=0$. This doesn't change the structure of the horizon, and in particular, the RN black hole spacetime is still regular there. In the near horizon limit, one observes that

$$
\lim _{\rho \rightarrow 0}\left(1+\frac{r_{0}}{\rho}\right)^{-2}=\left(\frac{r_{0}}{\rho}\right)^{-2} \text { and } \lim _{\rho \rightarrow 0}\left(1+\frac{r_{0}}{\rho}\right)^{2}=\left(\frac{r_{0}}{\rho}\right)^{2}
$$

so that the geometry in the near-horizon limit is given as

$$
d s^{2}=-\left(\frac{r_{0}}{\rho}\right)^{-2} d t^{2}+\left(\frac{r_{0}}{\rho}\right)^{2} d \rho^{2}+r_{0}^{2} d \Omega^{2} .
$$

By setting $r=\frac{r_{0}^{2}}{\rho}$ and up to redefinition, one obtain the near horizon geometry as

$$
d s^{2}=\left(\frac{r_{0}}{r}\right)^{2}\left(-d t^{2}+d r^{2}\right)+r_{0}^{2} d \Omega^{2} .
$$

Along the $r$ - $t$ direction, one sees that the geometry has negative curvature, while the angular direction has a geometry of a two sphere $S^{2}$ of radius $r_{0}^{2}$. As such, the geometry of RN black holes in the near-horizon limit is $A d S_{2} \times S^{2}$. Later, we shall see how near horizon solution arise from the higher dimensional configurations of supergravity theory.

\subsubsection{Spacetime with a cosmological constant}

The simplest example of asymptotically (A)dS black hole is the Kottler solution [5], also known as the Schwarzschild-(A)dS solution. In the presence of $\Lambda$, the solution (2) receives a modification leading to the metric

$$
d s^{2}=-\left(1-\frac{\mu}{r^{d-3}}-\frac{2 \Lambda}{(d-1)(d-2)} r^{2}\right) d t^{2}+\frac{d r^{2}}{1-\frac{\mu}{r^{d-3}}-\frac{2 \Lambda}{(d-1)(d-2)} r^{2}}+r^{2} d \Omega_{d-2}^{2} .
$$

This spacetime solution is

$$
\left\{\begin{array}{lll}
\text { Schwarzschild-AdS } & \text { for } & \Lambda<0 \\
\text { Schwarzschild } & \text { for } & \Lambda=0 \\
\text { Schwarzschild-dS } & \text { for } & \Lambda>0 .
\end{array}\right.
$$


This class of solution has regular horizon for any mass parameter $\mu>0$. In $d=4$, the Kretschmann invariant for the Schwarzschild-(A)dS solution is given as

$$
R_{\mu \nu \rho \sigma} R^{\mu \nu \rho \sigma}=\frac{48 G^{2} M^{2}}{r^{6}}+\frac{8 \Lambda^{2}}{3},
$$

indicating that the solutions posses a real spacetime singularity at $r=0$. It has been shown that this solution is stable against linearized gravitational perturbation [10].

In the limit of vanishing mass of solution (14), one can obtain an ordinary (anti) de-Sitter spacetime of constant (negative) positive curvature. The simplest (A)dS spacetime is the maximally symmetric spacetime solution of the vacuum Einstein equations with a cosmological constant.

The $d$-dimensional de Sitter space $\mathrm{dS}_{d}$ can be viewed as a hyperbloid

$$
-X_{0}^{2}+X_{1}^{2}+\ldots .+X_{d}^{2}=\ell^{2}
$$

embedded in $(d+1)$-dimensional Minkowski spacetime $\mathbb{R}^{1, d}$, where $\ell$ is the radius of $\mathrm{dS}_{d}$. The metric in the embedding space $\mathbb{R}^{1, d}$ when expressed in global coordinate, $X_{0}=\ell \sinh (t / \ell), X_{i}=$ $\ell \omega_{i} \cosh (t / \ell)$, with $\omega_{i}$ constrained within a unit sphere $\vec{\omega}=1$ is given as

$$
d s^{2}=-d t^{2}+\ell^{2} \cosh ^{2}(t / \ell) d \Omega_{d-1}^{2} .
$$

This coordinate covers the entire hyperbloid and so, it is global. The geometry of $\mathrm{dS}_{d}$ in the static coordinate is

$$
d s^{2}=-\left(1-\frac{r^{2}}{\ell^{2}}\right) d t^{2}+\left(1-\frac{r^{2}}{\ell^{2}}\right)^{-1} d r^{2}+r^{2} d \Omega_{d-2}^{2},
$$

solving the vacuum EFE with positive cosmological constant which is related to the $\mathrm{dS}_{d}$ radius by

$$
\Lambda=\frac{(d-1)(d-2)}{2 \ell^{2}}
$$

The geometry of an ordinary $\mathrm{AdS}_{d}$ in the static coordinate is

$$
d s^{2}=-\left(1+\frac{r^{2}}{\ell^{2}}\right) d t^{2}+\left(1+\frac{r^{2}}{\ell^{2}}\right)^{-1} d r^{2}+r^{2} d \Omega_{d-2}^{2},
$$

which solves the vacuum EFE with negative cosmological constant $\Lambda=-\frac{(d-1)(d-2)}{2 \ell^{2}}$. While the negative cosmological constant is incompatible with our present universe, spacetimes with negative cosmological constant have some features that allow for an interesting duality between string theory on spacetimes asymptotic to $\mathrm{AdS}_{n} \times X^{d-n}$, where $X^{d-n}$ is a compact manifold, and conformal field theory (CFT) defined on the conformal boundary of $\mathrm{AdS}_{n}$ - this is the the AdS/CFT correspondence. This duality is the starting motivation of this lecture.

\section{$1.2 \quad$ Extended Objects: $p$-Branes}

We started with the concept of black hole as a point-like object that enjoys $S O(1,3)$ symmetry. Furthermore, we recollect that the starting philosophy of string theory is underlay by the generalization of the concept of zero-dimensional point particle in the standard QFT to a onedimensional string. In fact. these concepts can be generalized to describe the geometry and 
fields corresponding to $(p+1)$-dimensional objects that sweep out a certain worldvolume. They are regarded as $p$-branes. When these extended objects feature the event horizon, expecially in $d>4$, they represent the higher dimensional analog of black holes. The important role of D-branes is in its appearance as solitons for probing the non-perturbative properties of string theories. The generic picture of $\mathrm{D} p$-brane is depicted in figure 1 .

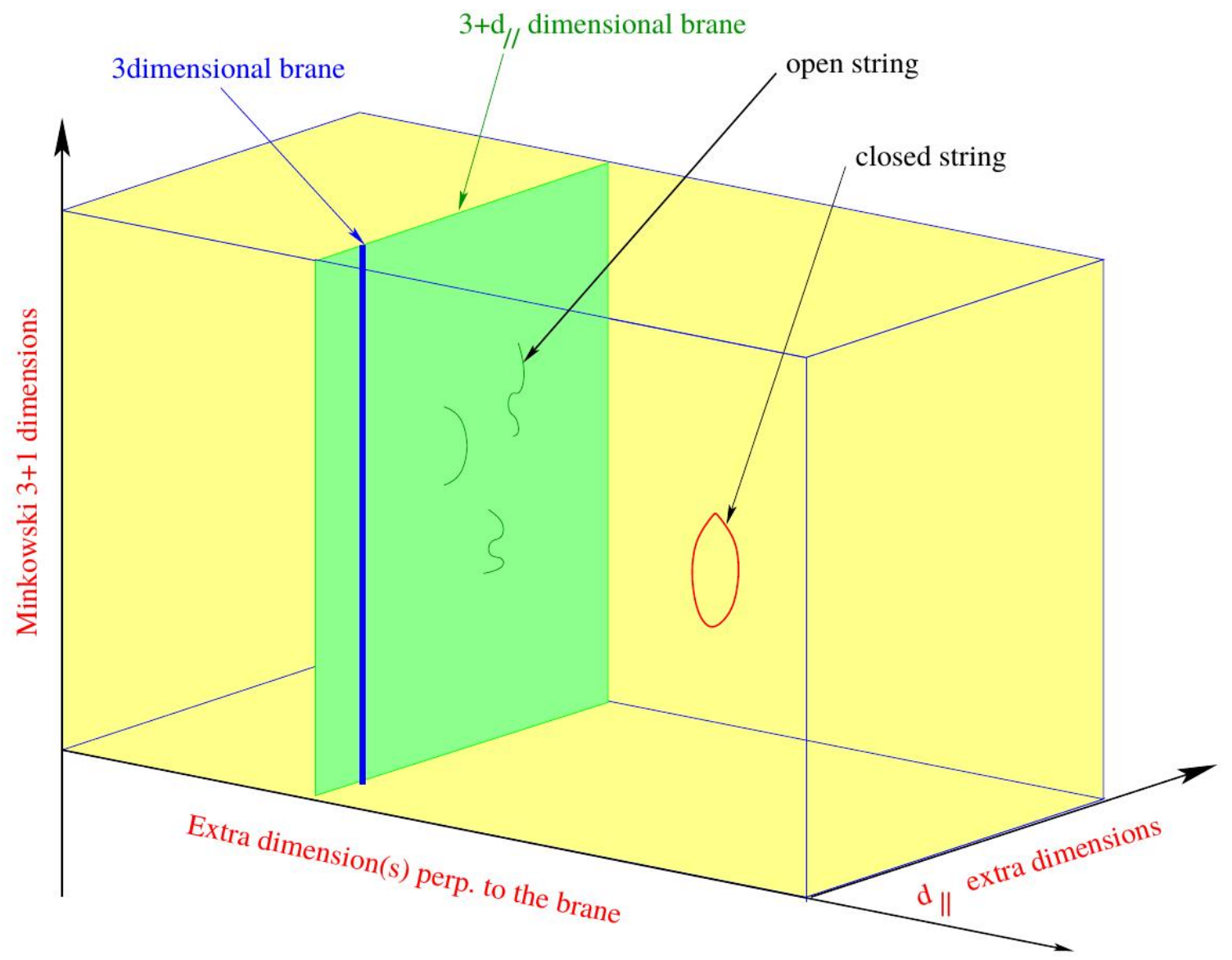

Figure 1. D-brane configuration featuring our $(3+1)$-dimensional world alongside $d_{\|}$parallel and $d_{\perp}$ tranverse internal directions [11].

As we shall see, these configurations arise as the classical solutions to various effective supergravity theory and their presence would break the initial lorentzian symmetry from

$$
S O(1, d-1) \rightarrow S O(1, p) \times S O(d-p-1),
$$

where $S O(1, p)$ describes the lorentz symmetry along the brane and $S O(d-p-1)$ along the direction perpendicular (transverse) to the brane.

p-brane configuration of supergravity theories feature various AdS geometries in their near horizon limits. Asymptotically $\mathrm{AdS}_{d}$ black hole solutions of the $d=4,5,6,7$ gauged supergravity theories also arises from dimensional reduction of $d=10$ or $d=11$ supergravity on spheres. Along the way, one finds that by replacing D-brane and open strings by their gravitational cloud, one obtain an analogous black hole physics. This represents the origin of the connection between string theory and black hole physics and has motivated many exciting topics. 


\section{Elements of Supergravity}

Supergravity is now over 40 year old into its development [12]. So, we shall not attempt to provide a full detail into its constructions. Interested readers can consult the reviews in $[13,14]$. Rather, we shall provide a brief introduction to supergravity, and then focus on 10D and 11D supergravity theories, which respectively provide the low energy effective field theory of the massless sector of superstring theory and M-theory.

\subsection{Supergravity: Reminder}

Supergravity (SUGRA) is simply a supersymmetric extension of ordinary classical gravity theory, so that the least of such extension unfies GR and supersymmetry in a consistent way. Supersymmetry (SUSY) on itself was born out of curiosity of finding a stabilisation mechanism to resolve the "hierarchy problem", and as it turned, the possible resolution led to a spacetime symmetry that exhanges boson for fermions. Schematically,

$$
\begin{aligned}
\mathcal{Q} \mid \text { Boson }\rangle & =\mid \text { Fermion }\rangle \\
\mathcal{Q} \mid \text { Fermion }\rangle & =\mid \text { Boson }\rangle,
\end{aligned}
$$

where $\mathcal{Q}$ is the SUSY fermionic generator i.e. they are spacetime spinors. As $\mathcal{Q}$ changes the spin of particles, it ultimately changes the properties of spacetime. Thus, SUSY is a spacetime symmetry that extends Poincare symmetry and its algebra. The structure of super-algebra was explored to circumvent the classical no-go theorems that constrain any possibility of unifying spacetime symmetries with internal symmetry. In particular, $\mathcal{Q}$ commutes with translation generator $P_{\mu}$ and internal symmetry $\mathcal{G}$ (e.g. gauge and global symmetries), but it does not commute with Lorentz generators $M_{\mu \nu}$. Schematically,

$$
\left[\mathcal{Q}, P_{\mu}\right]=0, \quad[\mathcal{Q}, \mathcal{G}]=0, \quad\left[\mathcal{Q}, M_{\mu \nu}\right] \neq 0
$$

and the anticommutation relation is $\{\mathcal{Q}, \overline{\mathcal{Q}}\} \sim P_{\mu}$. As such, the most general symmetry group of the S-matrix can enjoy is

\section{SuperPoincaré $\times$ Internal Symmetries}

Obviously, Poincaré symmetry is a subset of the SuperPoincaré symmetry. Aside from the (Poincaré $\times$ Internal Symmetries) algebra, the SUSY algebra includes the following:

$$
\begin{gathered}
{\left[M_{\mu \nu}, \mathcal{Q}_{\alpha}^{I}\right]=i\left(\sigma_{\mu \nu}\right)_{\alpha}^{\beta} \mathcal{Q}_{\beta}^{I},} \\
\left\{\mathcal{Q}_{\alpha}^{I}, \mathcal{Q}_{\beta}^{J}\right\}=\epsilon_{\alpha \beta} Z^{I J}, \quad Z^{I J}=-Z^{J I}, \\
\left\{\mathcal{Q}_{\alpha}^{I}, \overline{\mathcal{Q}}_{\dot{\beta}}^{J}\right\}=2 \sigma_{\alpha \dot{\beta}}^{\mu} P_{\mu} \delta^{I J},
\end{gathered}
$$

where $Z^{I J}$ is called the central charge and the index $I=\{1, . ., k\}$ for $\mathcal{N}=k$ SUSY.

According to Wigner classification, particles are nothing but the irredcible representation of the Poincaré group. Based on the Casimir of Poincaré algebra, such representations are constructed in ordinary quantum mechanics. In SUSY, the Wigner classification is combined with the structure of SUSY algebra to construct SUSY representations. Here, we shall not linger on such construction, and interested readers are encouraged to exercise through the construction of these representations. Hint on how to construct them are found in, for example $[13,14,15,16]$. 
What is however important to take along is that, in a model where SUSY is realized, each 1particle state in the supermultiplet has at least one superpartner related to it by SUSY generator and thus, one only deal with supermultiplets of particle states in a SUSY universe. As such, (24) implies that particles belonging to the same supermultiplet have different spin/helicity but same mass/energy and internal quantum numbers. The difference in spin/helicity can be straightforwardly understood from (25) since $\left[M_{12}, \mathcal{Q}_{2}^{I}\right]=-\frac{1}{2} \mathcal{Q}_{2}^{I}$ and $\left[M_{12}, \overline{\mathcal{Q}}_{\dot{2}}^{I}\right]=\frac{1}{2} \mathcal{Q}_{\dot{2}}^{I}$, implying that operator $\mathcal{Q}_{2}^{I}$ and $\overline{\mathcal{Q}}_{\dot{2}}^{I}$ lower, respectively raise, the helicity (spin) by half unit. In table 1 , we collect the massless supergravity multiplet corresponding to some extended supergravity theories i.e beyond $\mathcal{N}=1$ SUGRA.

\begin{tabular}{|l||l||l||l||l||l||l||l||l|}
\hline \multicolumn{7}{|c|}{ Contents of Supergravity Multiplets } \\
\hline$h$ & $\mathcal{N}=1$ & $\mathcal{N}=2$ & $\mathcal{N}=3$ & $\mathcal{N}=4$ & $\mathcal{N}=5$ & $\mathcal{N}=6$ & $\mathcal{N}=7$ & $\mathcal{N}=8$ \\
\hline+2 & 1 & 1 & 1 & 1 & 1 & 1 & 1 & 1 \\
\hline$+\frac{3}{2}$ & 1 & 2 & 3 & 4 & 5 & 6 & $7+1$ & 8 \\
\hline+1 & & 1 & 3 & 6 & 10 & $15+1$ & $21+7$ & 28 \\
\hline$+\frac{1}{2}$ & & & 1 & 4 & $10+1$ & $20+6$ & $35+21$ & 56 \\
\hline 0 & & & & $1+1$ & $5+5$ & $15+15$ & $35+35$ & 70 \\
\hline$-\frac{1}{2}$ & & & 1 & 4 & $10+1$ & $20+6$ & $35+21$ & 56 \\
\hline-1 & & 1 & 3 & 6 & 10 & $1+15$ & $7+21$ & 28 \\
\hline$-\frac{3}{2}$ & 1 & 2 & 3 & 4 & 5 & 6 & $7+1$ & 8 \\
\hline-2 & 1 & 1 & 1 & 1 & 1 & 1 & 1 & 1 \\
\hline
\end{tabular}

Table 1. Massless Supergravity representations for $d=4,[17]$.

As one may notice, beyond $\mathcal{N}=1$, supergravities generally contain vector fields $A_{\mu}$, spinor fields $\psi$ and scalar $\phi$ fields in addition to the gravitational field $e_{a}^{\mu}$ and a field $\Psi_{a}^{\mu}$ corresponding to spin$3 / 2$ particle. In order to couple gravity to spinor, the use of the veilbein, $e_{a}^{\mu}(a=0,1, \ldots, d-1)$, representing the gravitational field becomes necessary in some formulation. The metric is related to the vielbein as

$$
g_{\mu \nu} \equiv e_{\mu}^{a}(x) e_{\nu}^{b}(x) \eta_{a b}
$$

and we choose $e_{\mu}^{a}$ such that $e=\sqrt{-g}$.

In SUSY, the variation of a generic field $\Phi(x)$ is given as

$$
\delta_{\mathcal{Q}}(\epsilon) \Phi(x)=-i\left[\bar{\epsilon}^{\alpha} \mathcal{Q}_{\alpha}, \Phi(x)\right]
$$

and as a consequence, the commutator of two SUSY variation gives

$$
\begin{aligned}
{\left[\delta_{\mathcal{Q}}\left(\epsilon_{1}\right), \delta_{\mathcal{Q}}\left(\epsilon_{2}\right)\right] \Phi(x) } & =\left[\bar{\epsilon}_{1} \mathcal{Q},\left[\overline{\mathcal{Q}}_{2}, \Phi(x)\right]\right]-\left(\epsilon_{1} \leftrightarrow \epsilon_{2}\right), \\
& =\bar{\epsilon}_{1}^{\alpha}\left[\left\{\mathcal{Q}_{\alpha}, \overline{\mathcal{Q}}_{\dot{\beta}}\right\}, \Phi(x)\right] \epsilon_{2}^{\beta}, \\
& =\frac{1}{4} \bar{\epsilon}_{1} \gamma^{\mu} \epsilon_{2} \partial_{\mu} \Phi(x) .
\end{aligned}
$$

This is nothing but an infinitesimal spacetime translation with parameter $\frac{1}{4} \bar{\epsilon}_{1} \gamma^{\mu} \epsilon_{2}$.

In SUGRA, the spinorial SUSY parameter $\epsilon$ is promoted to a local gauged parameter $\epsilon(x)$, so that SUSY is gauged. Due to the spinorial nature of $\epsilon(x)$, the gauge field of the local SUSY is the Rarita-Schwinger field $\Psi_{a}^{\mu}$ representing a spin-3/2 field called gravitino. The Rarita-Schwinger field has two indices: the world index $\mu$ and the spinor index $a$. Just like in electromagnetic theory, the two form $\partial_{\mu} \Psi_{\nu}^{a}-\partial_{\nu} \Psi_{\mu}^{a}$ is a gauge invariant under the gauge transformation

$$
\Psi_{\mu}^{a} \rightarrow \Psi_{\mu}^{a}+\partial_{\mu} \epsilon^{a}(x) .
$$


From table 1, we already saw that the SUGRA multiplet of the $\mathcal{N}=1, D=4$ Poincaré supergravity theory consists of representation $\left(\frac{3}{2}, 2\right) \oplus\left(-2,-\frac{3}{2}\right)$ corresponding to a pair of fields $\left\{e_{a}^{\mu}, \Psi_{a}^{\mu}\right\}$. The basic element of SUGRA is encoded in the " 1.5 order formalism" :

$$
\begin{gathered}
S=\frac{1}{2 \kappa^{2}} \int d^{4} x e\left[e_{a}^{\mu} e_{b}^{\nu} R_{\mu \nu}{ }^{a b}(\omega)-\frac{1}{2} \bar{\Psi}_{\mu} \gamma^{\mu \nu \rho} D_{\nu}(\omega) \Psi_{\rho}\right] \\
\frac{\partial S}{\partial \omega}=0 \Longrightarrow D_{\mu} e_{a}^{\nu}-D_{\nu} e_{a}^{\mu}=\frac{1}{4} \bar{\Psi}_{\mu} \gamma^{a} \Psi_{\nu} \\
\delta_{\mathcal{Q}} e_{\mu}^{a}=\frac{1}{4} \bar{\epsilon} \gamma^{a} \Psi_{\mu}, \delta_{\mathcal{Q}} \Psi_{\mu}=D_{\mu} \epsilon
\end{gathered}
$$

where $\omega$ is the spin connection corresponding to the gauge field of the local lorentz transformation $\delta_{L}(\lambda)$ enjoyed by the theory. In addition, the theory is invariant up to total divergences under general coordinate transformation $\delta_{G}(\xi)$. Altogether, the local transformations satisfy, among others, the commutation relation

$$
\left[\delta_{\mathcal{Q}}\left(\epsilon_{1}\right), \delta_{\mathcal{Q}}\left(\epsilon_{2}\right)\right]=\delta_{G}(\xi)+\delta_{L}(\lambda)+\delta_{\mathcal{Q}}(\epsilon)
$$

where the transformation parameters are

$$
\xi^{\mu}=\frac{1}{4} \bar{\epsilon}_{2} \gamma^{\mu} \epsilon_{1} \quad, \quad \lambda_{a b}=-\xi_{\mu} \omega_{a b}^{\mu} \quad, \quad \epsilon=-\xi^{\mu} \Psi_{\mu} .
$$

The field equations under (32) are known to have Minkowski space preserving SUSY as a classical solution. Theories with this kind of solution are regarded as Poincaré supergravity. Matter and gauge multiplets can be coupled to the SUGRA multiplet, but this is not of our primary interest.

The field content of the SUGRA multiplet of the $(d, \mathcal{N})$ theory are listed in chapter 3 of [14]. The configurations of interest to us are those arising as the classical solution to 10D and 11D supergravity, which are known to respectively provide the low energy effective field theory of superstring theories and M-theory. Thus, we shall first collect the massless sectors of these theories. While we shall list their full particle spectrum, the bosonic sector will be the main content of the theory we wish to solve since the classical configurations arising from the corresponding SUGRA solution are purely bosonic.

Various ranks of anti-symmetric tensors field arise in SUGRA in higher dimension. As a consequence, further restriction on the corresponding field equation might arise. Without loss of generality, consider SUGRA theory featuring a rank $p$ gauge potential $A_{\mu_{1} \mu_{2} \ldots \mu_{p}}$. The corresponding field strength is given as

$$
F_{p}=F_{\mu_{1} \mu_{2} \ldots \mu_{p+1}}=(p+1) \partial_{\left[\mu_{1}\right.} A_{\left.\mu_{2} \ldots \mu_{p+1}\right]},
$$

and can be shown to be invariant under the gauge transformation $\delta A_{\mu_{1} \mu_{2} \ldots \mu_{p}}=p \partial_{\left[\mu_{1}\right.} \varepsilon_{\left.\mu_{2} \ldots \mu_{p}\right]}$, where $\varepsilon_{\mu_{1} \ldots \mu_{p-1}}(x)$ is the gauge parameter. The following are taken into consideration while studying SUGRA in higher dimension:

- SUGRA described by a gauge potential $A_{\mu_{1} \mu_{2} \ldots \mu_{p}}$ can be equivalently described by the dual gauge potential $\tilde{A}_{\mu_{1} \ldots \mu_{D-p-2}}$ since they have equal degrees of freedom.

- In even dimension $d=4 k+2$, self-duality equation

$$
F_{\mu_{1} \mu_{2} \ldots \mu_{2 k+1}}=\star F_{\mu_{1} \mu_{2} \ldots \mu_{2 k+1}}
$$

must be imposed on the field equation. $\star$ is the Hodge dual operator on the field strength. 


\section{BPS Property}

In an extended supersymmetric field theory, some extremal supermultiplets do retain some amount of supersymmetries. An important physical invariant to characterize this is the fraction

$$
\nu=\frac{\mathcal{N}_{Q}}{\mathcal{N}_{Q_{0}}},
$$

of supersymmetry that are preserved by supersymmetric vacuum solution, where $\mathcal{N}_{Q_{0}}$ is the total number of supercharges of an $\mathcal{N}=k$ SUSY and $\mathcal{N}_{Q}$ is the number of supercharges that are left unbroken by the solution.

Following (26), an $\mathcal{N}=k$ SUSY algebra contains central charges $Z$, which are generators commuting with any generator of the algebra. Consider $\mathcal{N}=2$ in $D=4$. We define

$$
\mathcal{Q}_{\alpha}^{ \pm}=\frac{1}{2}\left(\mathcal{Q}_{\alpha}^{1} \pm \epsilon_{\alpha \beta}\left(\mathcal{Q}_{\beta}^{2}\right)^{\dagger}\right)
$$

In the rest frame, $P_{\mu}=(M, 0,0,0)$, so that the algebra (27) becomes

$$
\left\{\mathcal{Q}_{\alpha}^{ \pm},\left(\mathcal{Q}_{\beta}^{ \pm}\right)^{\dagger}\right\}=\delta_{\alpha \beta}(M \pm|Z|)
$$

Now, a state of mass $M$ and of central charge $Z$ satisfies the inequality

$$
\begin{aligned}
0 & \leq \| \mathcal{Q}_{\alpha}^{-}|M, Z\rangle\left\|^{2}+\right\|\left(\mathcal{Q}_{\alpha}^{-}\right)^{\dagger}|M, Z\rangle \|^{2} \\
& =\left\langle M, Z\left|\left\{\mathcal{Q}_{\alpha}^{-},\left(\mathcal{Q}_{\beta}^{-}\right)^{\dagger}\right\}\right| M, Z\right\rangle \\
& =M-|Z|
\end{aligned}
$$

$$
|M, Z\rangle \text { saturates the Bogomol'nyi-Prasad-Sommerfield (BPS) bound } M \geq|Z| \text {. }
$$

This means that the mass of a given irreducible representation is always larger or equals to the eigenvalue of the central charge. We thus define a BPS state as a state $|M, Z\rangle$ satisfying the BPS bound $M=|Z|$.

When $\nu=1 / 2$, such solution saturates the so called Bogomol'nyi-Prasad-Sommerfield bound and are thus called the BPS bound state.

Whenever a state satisfies this requirement, such a state is annihilated by half SUSY i.e. $\mathcal{Q}_{\alpha}^{-}$, and form an irreducible representation under the remaining SUSY algebra. As a consequence, BPS states organize themselves into short multiplets as opposed to the long multiplets of the non-BPS state. Following this, supersymmetric vacuum solution that preserves $\nu=1 / 2$ SUSY saturates the BPS bound and are thus called the BPS bound state.

An important comment is in order. Suppose a state $|M, Z\rangle$ is BPS at weak coupling $g \ll$ 1 , renormalization group implies that both $M$ and $Z$ would recieve some form of corrections at strong coupling $g$, either perturbatively or non-perturbatively. Despite this correction, the number of state inside $|M, Z\rangle$ does not vary with $g$ and as such, short multiplet cannot become long multiplet along renormalization group flow. Thus, BPS property is preserved also at strong coupling regime. This property makes BPS object a very stable object.

$$
\text { If }|M, Z\rangle \text { is BPS at } g \ll 1 \text {, then }|M, Z\rangle \text { is BPS for all } g \text {. }
$$

We have already encountered a similar feature before, namely, the extremal limit of the RN black hole has the property that its mass is equivalent to its charge. Thus, violation of the 
BPS bound could translate to the appearance of naked singularity, which is forbidden by cosmic censorship. As we shall later see, the classical $p$-brane configurations that we shall construct are BPS. This makes these objects of physical relevance for studying the strong coupling regime of the corresponding higher dimensional theory.

\section{p-Brane Charge}

In section 1.2 , we introduced $p$-brane as a generalization of the concept of zero-dimensional point particle to a $(p+1)$-dimensional objects, sweeping out a certain worldvolume. Just like in the classical electromagnectic $\mathrm{U}(1)$ gauge theory, where particles are charged by the $\mathrm{U}(1)$ gauge field, the explicit $p$-brane solutions that we shall construct are charged under Abelian higher rank gauge fields. Thus, there ought to be a generalization of the Maxwell's theory to extended objects. The classical electromagnetism is described by the classical $U(1)$ gauge theory

$$
S=\int d^{4} x\left(-\frac{1}{2} F \wedge \star F-A \wedge \star J\right)
$$

where $J=J_{\mu} d x^{\mu}$ and $J_{\mu}=(\rho, \vec{j})$ is the 4-current. The EOM is simply the Maxwell field equation

$$
d \star F=\star J_{e} \quad, \quad d F=\star J_{m} .
$$

We can already see that, in vacuum, the theory is invariant under $F \rightarrow \star F$, i.e. $(\vec{E}, \vec{B}) \rightarrow$ $(\vec{B},-\vec{E})$. This is the electric-magnetic duality.

For a point-like electric source

$$
\rho_{e}=q_{e} \delta^{3}(\vec{r}) \quad \text { with electric charge } q_{e}=\int_{S^{2}} \star F,
$$

and its point-like magnetic dual source

$$
\rho_{m}=q_{m} \delta^{3}(\vec{r}) \quad \text { with magnetic charge } \quad q_{m}=\int_{S^{2}} F .
$$

Dirac quantization condition

$$
q_{e} \cdot q_{m} \in 2 \pi \mathbb{Z}
$$

is satisfied.

Thus, in higher dimension, an extended object with $(p+1)$-dimensional worldvolume would coupled electrically to a $(p+1)$-form gauge ptential. This is described by the action

$$
S=\int_{\mathcal{M}} d^{d} x\left(-\frac{1}{2} F_{p+2} \wedge \star F_{p+2}-A_{p+1} \wedge \star J_{p+1}^{e}\right),
$$

and one finds the electric charge

$$
Q_{e}=(-1)^{p} \int_{S^{d-p-2}} \star F_{p+2} .
$$

The magnetic dual object, which sweep out a $(d-p-3)$-dimensional worldvolume, corresponds to a $(d-p-4)$-brane coupling magnetically to a $(d-p-3)$-form gauge potential. The corresponding magnetic charge is given as

$$
Q_{m}=\int_{S^{p+2}} F_{p+2},
$$

and the Dirac quantization has a straightforward generalization $Q_{e} \cdot Q_{m} \in 2 \pi \mathbb{Z}$. 


\subsection{Superstring/M-theory: Towards 10D and 11D SUGRA}

There are 5 manifestation of superstring theories, all consistent in 10 spacetime dimensions and related by various string dualities (cf. Figure 2). String duality has also led to the prediction of an $11 D$ theory called M-theory. The classical limit of superstring theories and M-theory are respectively given by the $10 D$ and $11 D$ supergravity theories. The later are the theories whose classical solution we demand. Thus, we shall first collect the massless sectors of these theories towards providing an effective field theories describing them. Towards our study on $p$-brane solutions which are purely bosonic extended objects, we shall provide the supergravity action for only the bosonic content, and discuss less of the corresponding string dynamics. In this case, we maintain $G_{M N}=e_{M}^{a} e_{N}^{b} \eta_{a b}$ as the carrier of the gravitational field.

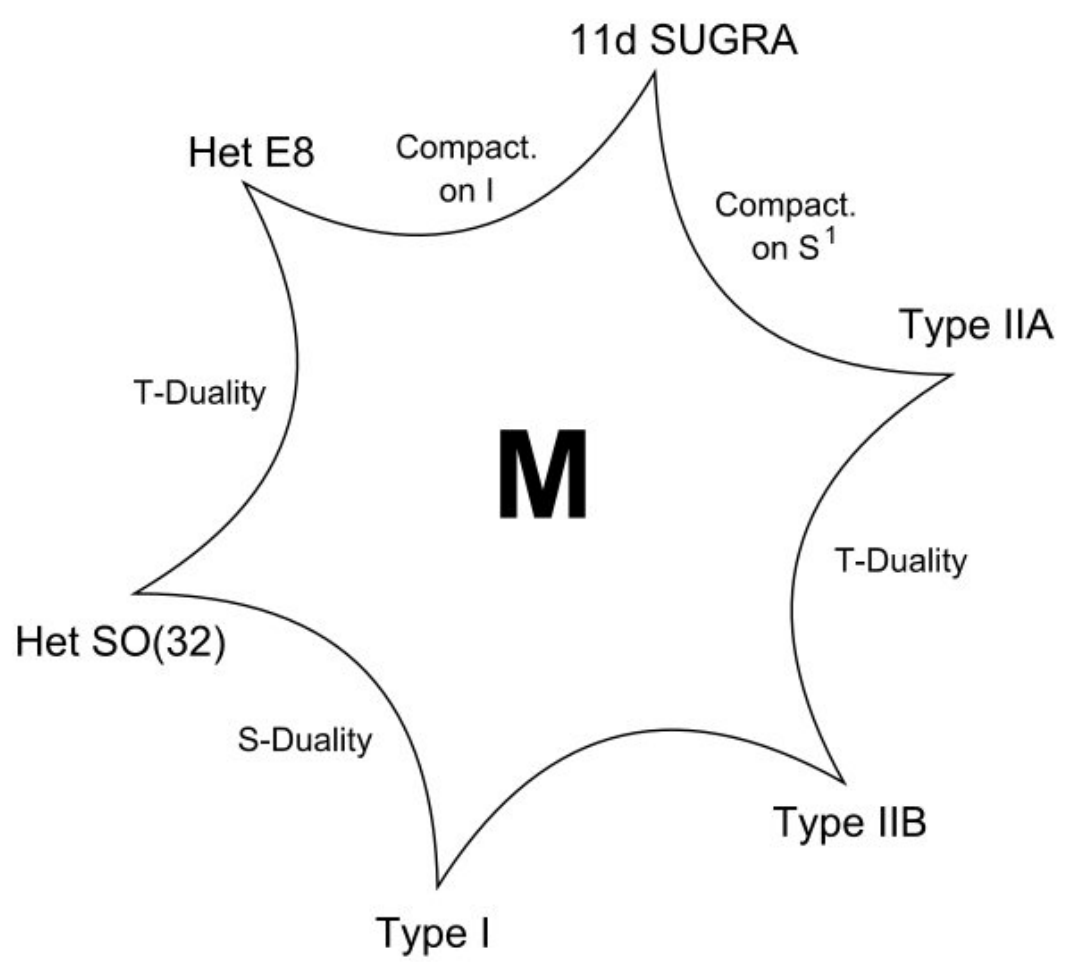

Figure 2. Various String theories related by string dualities - Wiki

\subsubsection{Massless Sectors of Superstring Theories}

In the following, we provide the massless sector of superstring theories. The spectrum are those of the corresponding $10 \mathrm{D}$ supergravity theories. We denote the fermionic field chiralities by the subscript \pm . The superscript \pm on the antisymmetric tensor fields denotes the (anti-)self-duality.

1. Non-chiral Type IIA Superstring Theory: This is a $10 D$ theory of closed strings whose low energy effective theory gives the $d=10, \mathcal{N}=(1,1)$ supergravity (maximal SUSY with 32 
supercharges). The massless field content is

$$
\begin{gathered}
\text { NS-NS Sector: } G_{M N}, B_{M N}, \Phi, \\
\text { R-R Sector: } C_{M N}, C_{M N P}, \\
\text { NS-R, R-NS Sectors: } \Psi_{M+}, \Psi_{M-}, \lambda_{+}, \lambda_{-} .
\end{gathered}
$$

The leading order supergravity action of the bosonic sector is given by

$$
\begin{aligned}
S= & \frac{1}{2 \kappa_{10}^{2}} \int d^{10} x \sqrt{-G}\left\{e^{-2 \Phi}\left(R+4(\nabla \Phi)^{2}-\frac{1}{2}\left|H_{3}\right|^{2}\right)-\frac{1}{2}\left|F_{2}\right|^{2}-\frac{1}{2}\left|F_{4}\right|^{2}\right\} \\
& -\frac{1}{4 \kappa_{10}^{2}} \int B_{2} \wedge F_{4} \wedge F_{4},
\end{aligned}
$$

with gravitational coupling $\kappa_{10}^{2}=\frac{1}{4}\left(4 \pi^{2} \alpha^{\prime}\right)^{4}$. We have used the notation $\left|F_{p}\right|^{2}=\frac{1}{p !} F_{M_{1} \ldots M_{p}} F^{M_{1} \ldots M_{p}}$.

2. Chiral Type IIB Superstring Theory: This is a $10 D$ theory of closed strings whose low energy effective theory gives the $d=10, \mathcal{N}=(2,0)$ supergravity (maximal SUSY with 32 supercharges). The massless field content is

$$
\begin{aligned}
& \text { NS-NS Sector: } G_{M N}, B_{M N}, \Phi, \\
& \text { R-R Sector: } C, C_{M N}, C_{M N P Q}^{(+)},
\end{aligned}
$$

NS-R, R-NS Sectors: $2 \Psi_{M+}, 2 \lambda_{-}$.

The leading order supergravity action of the bosonic sector is given by

$$
\begin{aligned}
S= & \frac{1}{2 \kappa_{10}^{2}} \int d^{10} x \sqrt{-G}\left\{e^{-2 \Phi}\left(R+4(\nabla \Phi)^{2}-\frac{1}{2}\left|H_{3}\right|^{2}\right)-\frac{1}{2}\left|F_{1}\right|^{2}-\frac{1}{2}\left|F_{3}\right|^{2}-\frac{1}{2}\left|F_{5}\right|^{2}\right\} \\
& -\frac{1}{4 \kappa_{10}^{2}} \int C_{4} \wedge H_{3} \wedge F_{3} .
\end{aligned}
$$

3. Type I Superstring Theory: This is a $10 D$ theiry of closed and open strings whose low energy effective theory gives the $d=10, \mathcal{N}=(1,0)$ supergravity (with 16 supercharges) coupled to Super Yang-Mill theory with gauge group $G=S O(32)$. The massless field content is

$$
\begin{array}{r}
\text { Closed NS-NS Sector: } G_{M N}, \Phi, \\
\text { Closed R-R Sector: } C_{M N}, \\
\text { Closed NS-R, R-NS Sectors: } \Psi_{M+}, \lambda_{-}, \\
\text {Open NS Sector: } A_{M}, \\
\text { Open R Sector: } \chi_{+} .
\end{array}
$$

The leading order supergravity action of the bosonic sector is given by

$$
\begin{aligned}
S= & \frac{1}{2 \kappa_{10}^{2}} \int d^{10} x \sqrt{-G}\left\{e^{-2 \Phi}\left(R+4(\nabla \Phi)^{2}\right)-\frac{1}{2}\left|F_{3}\right|^{2}\right\} \\
& -\frac{1}{2 g_{10}^{2}} \int d^{10} x \sqrt{-G} e^{-\Phi} \operatorname{Tr}\left|F_{Y M}\right|^{2} .
\end{aligned}
$$

The field strength of the R-R antisymmetric 2-form receives additional contributions

$$
F_{3}=d C_{2}-\frac{\alpha^{\prime}}{4}\left(\Omega_{Y M}-\Omega_{L}\right)
$$


where $\Omega_{Y M}$ and $\Omega_{L}$ are the Yang-Mills and Lorentz the Chern-Simons 3-forms

$$
\begin{aligned}
\Omega_{Y M} & =\operatorname{Tr}\left(A \wedge d A-\frac{2 i}{3} A \wedge A \wedge A\right), \\
\Omega_{L} & =\operatorname{Tr}\left(A \wedge d A-\frac{2 i}{3} \omega \wedge \omega \wedge \omega\right),
\end{aligned}
$$

and $\omega$ is the spin connection. $F_{Y M}=F_{Y M}^{a} T^{a}$ is the Yang-Mills field strength and the trace is over the vector representation of the gauge group with $\operatorname{Tr}\left(T^{a} T^{b}\right)=\delta^{a b}$.

4. Heterotic String theory: There are two Heterotic string theories consistent in $10 D$ describing closed strings, whose effective field theory is given by $d=10, \mathcal{N}=(1,0)$ supergravity (with 16 supercharges) coupled to Super Yang-Mill theory with gauge group $G=S O(32)$ or $E_{8} \times$ $E_{8}$. The massless field content is

$$
\begin{aligned}
& \text { NS Sector: } G_{M N}, B_{M N}, \Phi, A_{M}, \\
& \text { R Sector: } \Psi_{M+}, \lambda_{-}, \chi_{+} .
\end{aligned}
$$

The supergravity action of the bosonic sector in the leading order is given by

$$
S=\frac{1}{2 \kappa_{10}^{2}} \int d^{10} x \sqrt{-G} e^{-2 \Phi}\left(R+4(\nabla \Phi)^{2}-\frac{1}{2}\left|H_{3}\right|^{2}\right)-\frac{1}{2 g_{10}^{2}} \int d^{10} x \sqrt{-G} e^{-2 \Phi} \operatorname{Tr}\left|F_{Y M}\right|^{2} .
$$

The field strength of the NS-NS antisymmetric 2-form receives contributions

$$
H_{3}=d B_{2}-\frac{\alpha^{\prime}}{4}\left(\Omega_{Y M}-\Omega_{L}\right) .
$$

\subsubsection{Massless Sectors of M-theory}

M-theory is an $11 D$ theory whose low energy effective field theory is given by $d=11, \mathcal{N}=1$ supergravity (maximal SUSY with 32 supercharges). The massless field content is

$$
G_{M N}, C_{M N P}, \Psi_{M}
$$

The action of the bosonic sector of the 11-dimensional supergravity is given as

$$
2 \kappa_{11}^{2} S=\int d^{11} x \sqrt{-G}\left(R-\frac{1}{2}\left|F_{4}\right|^{2}\right)-\frac{1}{6} \int C_{3} \wedge F_{4} \wedge F_{4},
$$

where $\kappa_{11}$ denotes the $11 D$ gravitational coupling.

\section{The $p$-Brane Solution}

We noted earlier on that the extended object with $(p+1)$-dimensional worldvolume would coupled electrically to a $(p+1)$-form gauge ptential and magnetically to a $(d-p-3)$-form gauge potential. This suggest that a $p$-brane solution to the classical field equation would necessarily contains a non-trivial $(p+1)$-form in the background configuration. The non-trivial classical solution is, thus, expected to be purely sourced by the energy momentum tensor of the $(p+1)$ form background. The extended charged configuration that we anticipate in superstring and M-theory can be explored by studying the various corresponding bosonic field contents. Brane configurations were first constructed in [18], with earlier review in [19, 20]. 
Without loss of generality, we begin by considering a bosonic string propagating in the background of its own massless excitation, namely $\left\{G_{M N}, B_{M N}, \Phi\right\}^{1}$. These are the sectors that couple directly with the string worldsheet. This is well described by a non-linear $\sigma$-model - a natural generalization of the closed Polyakov action [21, 22]

$$
S_{\sigma}=\frac{1}{4 \pi \alpha^{\prime}} \int_{\Sigma} d^{2} \xi \sqrt{-h}\left\{\left(h^{a b} G_{M N}(X)+i \epsilon^{a b} B_{M N}\right) \partial_{a} X^{M} \partial_{b} X^{N}+\alpha^{\prime} R^{(2)} \Phi(X)\right\} .
$$

$R^{(2)}$ is scalar curvature of the $2 \mathrm{D}$ worldsheet and $\Phi(X)$ is the background dilaton field of spacetime. The last term is the generalization of the topological term $\frac{1}{4 \pi} \int d^{2} \xi \sqrt{-h} R^{(2)} \lambda$ with $\lambda=\Phi(X)$. Thus, the string coupling is given as

$$
g_{s}=e^{\Phi}=e^{\Phi(X)},
$$

suggesting that the string coupling is dynamical.

String theory is not consistent in just any spacetime background, rather, it is consistent in those background that satisfies some required conditions on the worldsheet, namely the cancellation of anomaly in the local worldsheet symmetries. In particular, the $\sigma$-model is classically invariant under the worldsheet Weyl symmetry $h_{a b} \rightarrow e^{2 \Lambda(\xi)} h_{a b}$ whose consequence is the tracelessness of the energy momentum tensor i.e. $T_{a}^{a}=0$. However, this invariance is broken at quantum level by the dilaton part of the action (61) since there are ovarall non-zero contributions to the $\beta$-function such that scale invariance is lost. As a consequence, the corresponding $T_{a}^{a}$ vanishes classically but proportional to the non-vanishing $\beta$-function at the quantum level. The general structure of the trace is given as

$$
2 \pi T_{a}^{a}=\beta^{\Phi} \sqrt{-h} R^{(2)}+\beta_{M N}^{G} \sqrt{-h} h^{a b} \partial_{a} X^{M} \partial_{b} X^{N}+\beta_{M N}^{B} \epsilon^{a b} \partial_{a} X^{M} \partial_{b} X^{N}
$$

where $\beta^{\Phi}, \beta^{G}$ and $\beta^{B}$ are 1-loop $\beta$-functions associated with $\Phi, G_{M N}$ and $B_{M N}$ respectively. They are given as

$$
\begin{aligned}
& \beta_{M N}^{G}=\alpha^{\prime} R_{M N}+2 \alpha^{\prime} \nabla_{M} \nabla_{N} \Phi-\frac{\alpha^{\prime}}{4} H_{M P Q} H_{N}^{P Q}+\mathcal{O}\left(\alpha^{\prime 2}\right), \\
& \beta_{M N}^{B}=-\frac{\alpha^{\prime}}{2} \nabla^{P} H_{P M N}+\alpha^{\prime} \nabla^{P} \Phi H_{P M N}+\mathcal{O}\left(\alpha^{\prime 2}\right), \\
& \beta_{M N}^{\Phi}=\frac{D-26}{6}-\frac{\alpha^{\prime}}{2} \nabla^{2} \Phi+\alpha^{\prime} \nabla_{P} \Phi \nabla^{P} \Phi-\frac{\alpha^{\prime}}{24} H_{M N P} H^{M N P}++\mathcal{O}\left(\alpha^{\prime 2}\right) .
\end{aligned}
$$

Thus, requiring the anomalies cancellation of the Weyl symmetry at the quantum level lead to a system of coupled effective equations $\beta_{M N}^{G}=\beta_{M N}^{B}=\beta_{M N}^{\Phi}=0$ as restrictions on the background fields. The corresponding coupled equations are captured by an effective field theory with action

$$
S_{e f f}=\frac{1}{\kappa_{d}^{2}} \int d^{d} x \sqrt{-G} e^{-2 \Phi}\left[(d-26)-\frac{3}{2} \alpha^{\prime}\left(R+4 \nabla_{M} \Phi \nabla^{M} \Phi-4(\nabla \Phi)^{2}\right)-\frac{1}{12} H_{M N P} H^{M N P}\right] .
$$

This action already depicts the critical dimension 26 as a requirement for the consistency of the worldsheet theory of the bosonic string. One would arive at a effective action for superstring theories, with the replacement of the bosonic criticality by $(d-10)$ criticality term of the superstring theories.

The appearance of the prefactor $e^{-2 \Phi}$ shows that the effective action (65) is in string frame. By making a Weyl rescaling $G_{M N} \rightarrow e^{a_{p} \Phi} G_{M N}$ and up to relabelling, this action can be written in

\footnotetext{
${ }^{1}$ These are the NS-NS sectors in superstring models.
} 
the Einstein frame. Choosing $a_{p}=1 / 2$, we obtain the Einstein frame effective action

$$
S_{e f f}=\frac{1}{\kappa_{10}^{2}} \int d^{10} x \sqrt{-G}\left[R-\frac{1}{2} \nabla_{M} \Phi \nabla^{M} \Phi-\frac{1}{12} e^{\Phi} H_{M N P} H^{M N P}\right] .
$$

As we have seen in section (2.2.1), there are further bosonic fields in the R-R sectors, namely the various antisymmetric tensor gauge fields of various ranks. These must be taken into account while considering solving 10D and 11D supergravity field equations.

Following this, let us generalize into (66) into a classical supergravity system in an arbitrary $D$ dimensions featuring $\left\{G_{M N}, \Phi\right\}$ and a rank $(p+1)$ gauge potential $A_{p+1}$, with a corresponding field strength $F_{p+2}$. We shall drop the Chern-simon terms as it is a topological term. The low-energy effective action describing these excitations is

$$
S=\frac{1}{\kappa_{d}^{2}} \int d^{d} x \sqrt{-G}\left[R-\frac{1}{2} G^{M N} \nabla_{M} \Phi \nabla_{N} \Phi-\frac{1}{2(p+2) !} e^{a_{p} \Phi} F_{p+2} \wedge \star F_{p+2}\right] .
$$

Thus, looking into this action, we can already extract the effective actions we presented in section (2.2.1). For example, dropping the dilaton and setting $d=11, p=2$, and $a_{p}=0$, one realize back the $11 \mathrm{D}$ supergravity action (60). For the effective supergravity theories from superstrings, one can realize (67) by following the splitting of dilaton $\Phi=\Phi_{0}+\Phi^{\prime}$ with $e^{\Phi_{0}} \equiv g_{s}$. The metric is then rescaled and $\Phi^{\prime}$ is relabelled as $\Phi$. In turn, for various 10D SUGRA, $a_{p}$ takes a specific value e.g. Including NS-NS 2 -form requires $a_{-1}=-1$ while the R-R sectors requires $a_{p}=(3-p) / 2$. Furthermore, we should note that the parameter $a_{p}$ controls the coupling of the dilaton with the field strength $F_{p+2}$. For type IIB theory, we must separately impose the self-dulaity condition $F_{5}=\star F_{5}$.

The field equations following (67) are

$$
\left\{\begin{array}{l}
\square \Phi=\frac{a_{p}}{2(p+2) !} e^{a_{p} \Phi} F^{2}, \\
\nabla_{M}\left(e^{a_{p} \Phi} F^{M M_{1} \ldots M_{p+1}}\right)=0, \\
R_{M N}=\frac{1}{2} \nabla_{M} \Phi \nabla_{N} \Phi+\frac{e^{a_{p} \Phi}}{2(p+2) !}\left(F_{M N}^{2}-\frac{(p+1)}{(d-2)(p+2)} G_{M N} F^{2}\right) .
\end{array}\right.
$$

We now seek for brane configuration satisfying the above field equations. The presence of such configuration would break the $S O(1, d-1)$ symmetry to (cf. (22))

$$
S O(1, d-1) \rightarrow S O(1, p) \times S O(d-p-1) .
$$

Let us begin by denoting the dimension of the $p$-brane worldvolume by $n=p+1$ and as a consequence, the dimension of the worldvolume of the dual object as $\tilde{n}=\tilde{p}+1=d-n-2=$ $d-p-3$, so that the brane configuration we seek enjoys the folllowing corresponding symmetries:

$$
\begin{array}{r}
p-\text { brane : }(\text { Poincaré })_{n} \times S O(d-n), \\
\text { Dual Object : (Poincaré) })_{\tilde{n}} \times S O(d-\tilde{n}) .
\end{array}
$$

Non-extremal solution to (68) would further break the Lorentz symmetry along the brane into a subgroup. For our basic study, we shall not seek for this kind of configurations. Rather, we shall seek for brane solutions that preserve some amount of SUSY, in particular, solutions that are BPS. These are the higher-dimensional analogs of the extremal and near-extremal ReissnerNordström black hole that was introduced in section 1.1. Obviously, these objects satisfy the 
BPS properties. Given the $p$-brane world symmetry that we demand, we shall split of the spacetime indices as follows:

$$
x^{M}=\left(x^{\mu}, y^{i}\right), \quad \text { with }\left\{\begin{array}{l}
\mu=0,1, \ldots, n-1, \\
i=1, \ldots, d-n .
\end{array}\right.
$$

The most general ansatz for a brane configuration at $y^{i}=0$ satisfying the field equations (68) and the symmetry requirement (69) is given as

$$
\left\{\begin{array}{l}
e^{\Phi}=e^{\Phi(r)} \\
d s^{2}=e^{2 A(r)} \eta_{\mu \nu} d x^{\mu} d x^{\nu}+e^{2 B(r)} \delta_{i j} d y^{i} d y^{j},
\end{array}\right.
$$

with $r=y^{i} y^{j} \delta_{i j}$. The field strength corresponding to the gauge potential the are

$$
F_{p+2}^{\mathrm{elec}}=\Xi_{e} \star e^{-a_{p} \Phi} \epsilon_{S^{d-p-2}}, \quad F_{p+2}^{\mathrm{mag}}=\Xi_{m} \star \epsilon_{S^{p+2}},
$$

where $\epsilon_{S^{d-p-2}}$ is the volume form of the $(d-p-2)$-sphere surrounding the $p$-brane, and $\Xi_{e}$ and $\Xi_{m}$ are proportional to the electric and magnetic charge of the brane. Subtituting the ansatz into the field equations, we thus have a system of coupled non-linear differential equations subject to the following boundary conditions:

$$
\lim _{r \rightarrow \infty} G_{M N}=\eta_{M N}, \quad \lim _{r \rightarrow \infty} \Phi(r)=0 .
$$

Even with the general ansatz and the boundary conditions, it is still very challenging to solve the coupled non-linear differential equation. Certain class of solution can be obtain from the requirement that the solution preserves half SUSY i.e. solutions are BPS. The BPS requirement lead to an extra condition

$$
n A+\tilde{n} B=0 .
$$

This futher requirement, when imposed on the general ansatz, relaxes the complexity of the field equations. Details into solving the system of coupled non-linear differential equations can be found in $[18,19,20]$. Here, we shall straightforwardly state the main result.

The classical solution to (68) is characterized by an harmonic function $H(r)$ and the number $N$ is introduced for the case of coincident brane solution, the large limit of which the brane solutions makes sense. The brane solution is expressed as follows:

$$
\begin{aligned}
& \left\{\begin{array}{l}
d s^{2}=H^{-\frac{4 \tilde{n}}{\Delta(d-2)}} \eta_{\mu \nu} d x^{\mu} d x^{\nu}+H^{-\frac{4 n}{\Delta(d-2)}}\left(d r^{2}+r^{2} d \Omega_{d-n-1}\right), \\
e^{\Phi}=H^{\frac{2 a_{p}}{\xi \Delta}}, \quad \text { with } \quad \xi= \begin{cases}+1, & \text { electric brane } \\
-1, & \text { magnetic brane },\end{cases} \\
F_{p+2}^{\mathrm{el}}=(-1)^{p d+1} \frac{2 N}{\sqrt{\Delta}} \alpha \tilde{n} \star\left(e^{-a_{p} \Phi} \epsilon_{S^{\tilde{n}+1}}\right), \quad F_{p+2}^{\mathrm{el}}=\frac{2 N}{\sqrt{\Delta}} \alpha n \epsilon_{S^{n+1}},
\end{array}\right.
\end{aligned}
$$

with

$$
H(r)=1+\frac{N \alpha}{r^{\tilde{n}}}(\tilde{n}>0), \Delta=a_{p}^{2}+\frac{2 n \tilde{n}}{d-2},
$$

where $\alpha$ is related to the brane tension $\tau$ as $^{2}$

$$
\alpha=\frac{2 \kappa_{d}^{2} \tau_{n-1}}{\tilde{n} \Omega_{\tilde{n}+1}} \frac{\Delta}{4} .
$$

\footnotetext{
${ }^{2}$ For $d=10$ and $d=11$ SUGRA, one can check that $\Delta=4$.
} 
For elementary/electric brane, the normalized conserved electric charge density is given as

$$
Q_{e} \equiv N q_{e}=\frac{(-1)^{p}}{\sqrt{2} \kappa_{d}} \int_{S^{\tilde{n}+1}} e^{a_{p} \Phi} \star F_{p+2}^{\mathrm{ele}}=\sqrt{2} \kappa_{n} N \tau_{p}
$$

while the normalized conserved magnetic charge density solitonic/magnetic brane is given as

$$
Q_{m} \equiv N q_{m}=\frac{1}{\sqrt{2} \kappa_{d}} \int_{S^{n+1}} F_{p+2}^{\mathrm{mag}}=\sqrt{2} \kappa_{n} N \tau_{\tilde{p}}
$$

As a consequnce, the tension of the elementary brane and its solitonic dual satisfy the Dirac quantization condition $Q_{e} \cdot Q_{m} \in \mathbb{Z}$ leading to

$$
\tau_{p} \cdot \tau_{\tilde{p}}=\frac{\pi}{\kappa_{d}^{2}} n, \quad \text { with } \quad n \in \mathbb{Z}
$$

At this point, few comments are in order. In particular, string theory has a rich spectrum of extended objects analogous to some supergravity $p$-brane solution.

- First, the validity of the $p$-brane solutions is in the weak string coupling limit. Thus, for a characteristic $p$-brane length scale $L$ set by $\alpha \sim N \tau_{p} \kappa_{n}^{2} \sim L^{d-p-3}$, the $p$-brane solutions are only valid for large $N$ i.e. for large number of coincidental branes.

- It has been shown that D-brane tension

$$
\tau_{p}=\frac{2 \pi}{g_{s} \ell_{s}^{p+1}},
$$

of the type II string theories satisfy the matching with the charge (80) of the electric $p$ brane, as well as the Dirac quantization condition (82) corresponding to $p$-brane solution with $n=1$. This suggests that, despite the fact that these two objects arise from different description of gravity, they are in fact the same object.

- As the classical $p$-brane solution are charged, one expect that there would be some sort of competition among forces existing between two $p$-branes, namely, there is attractive force due to various exchange of gravitons and dilatons between the branes, and repulsive force due to the exchange of the $(p+1)$-form. In type II string theories, these $A_{p+1}$ corresponds to the exchange of the R-R form. It has been shown in that these forces precisely cancel out, independent of the distance between the two branes. This is in consonant with the fact that the constructed configurations are BPS and thus, they are stable due to the equality between the brane tension and the charge $(\tau=Q)$.

- As the $p$-brane configuration we constructed are BPS i.e. preserves $\nu=1 / 2$ SUSY, they thus satisfy the BPS bound requirement mass $=\mid$ charges $\mid$, which in turn makes these object extremal $p$-brane solutions with just one horizon. Non-extremal $p$-brane solutions exist, and they satisfies mass $\geq \mid$ charges $\mid$. These solutions ultimately breaks all SUSY. As a higher dimensional Reissner-Nordström black holes, they have two horizons and there exist no naked singularities forbidden by the cosmic censorship hypothesis. Solution that features naked singularities i.e. mass $\leq \mid$ charges $\mid$, are thus forbbiden by the BPS requirement.

At this point, let us now collect some of the most simplest BPS brane solutions. 


\subsection{Extremal Supermembranes}

The 11-dimensional SUGRA has four kinds of solutions BPS solutions, namely the $p p$-waves [23], the Kaluza-Klein monopole [24, 25], and the two elementary extremal membrane solutions - the electric M2-brane [26] and the its magnetic dual M5-brane [27]. Here, we shall collect the elementary membrane solution of (60). In the following, the notation $x_{\|}$denotes the directions that are parrallel to the brane i.e. $x^{\mu}$, while $x_{\perp}$ corresponds to those directions that are perpendicular to the brane i.e. $y^{i}$. We should not forget that the metric solution is lorentzian in the former direction and euclidean in the later direction.

- The Elementary M2-brane: The membrane or the M2-brane solution is electrically sourced by the 4 -form flux and enjoys $\mathrm{SO}(1,2) \times \mathrm{SO}(8)$ symmetry. The geometry is given as

$$
\begin{aligned}
d s^{2} & =H(r)^{-\frac{2}{3}} d x_{\|}^{2}+H(r)^{\frac{1}{3}} d x_{\perp}^{2}, \\
F_{4} & =d x^{0} \wedge d x^{1} \wedge d x^{2} \wedge d H(r)^{-1},
\end{aligned}
$$

with

$$
H(r)=1+\frac{\alpha}{r^{6}} \quad \text { and } \quad \alpha=32 \pi^{2} \ell_{11}^{6} N
$$

- The Solitonic M5-brane: The fivebrane or the M5-brane solution is magnetic dual of the M2-brane. Thus, this solution is magnetically sourced by the corresponding dual form flux and further enjoys $\mathrm{SO}(1,5) \times \mathrm{SO}(5)$ symmetry. The geometry is given as

$$
\begin{aligned}
d s^{2} & =H(r)^{-\frac{1}{3}} d x_{\|}^{2}+H(r)^{\frac{2}{3}} d x_{\perp}^{2}, \\
F_{4} & =\star\left(d x^{0} \wedge d x^{1} \wedge \ldots \wedge d x^{5} \wedge d H(r)^{-1}\right),
\end{aligned}
$$

with

$$
H(r)=1+\frac{\alpha}{r^{3}} \quad \text { and } \quad \alpha=\pi \ell_{11}^{3} N
$$

One can show that the above two solutions have horizons at $r=0$ where curvature diverges in the geometry. This suggest that there exist a source at $r=0$ whose strength is proportional to the corresponding M-brane charge and in turn, the number $N$ of corresponding stacked M-brane.

\subsection{Extremal Dp-branes}

To construct the extremal D-brane solution, we require the type II SUGRA action. We shall not include the NS-NS 2-form since it vanishes. Thus, we are left with only the R-R $(p+1)$-forms and the corresponding field strength $F_{p+2}$ enters the SUGRA action as expected. The action in the string frame boils down to

$$
S=\frac{1}{\kappa_{10}^{2}} \int d^{10} x \sqrt{-G}\left[e^{-2 \Phi}\left(R+4(\nabla \Phi)^{2}\right)-\frac{1}{2} F_{p+2} \wedge \star F_{p+2}\right]
$$

Now we have non-trivial dialton $\Phi$ which must be solved simultaneously. This actions represents the type IIA SUGRA when $p$ is even and type IIA SUGRA when $p$ is odd. Furthermore, we 
should not forget that for the case $p=3$, self-duality equation $F_{5}=\star F_{5}$ must be imposed alongside the field equations. The geometry is given as

$$
\begin{aligned}
d s^{2} & =H(r)^{\frac{p-7}{8}} d x_{\|}^{2}+H(r)^{\frac{p+1}{8}} d x_{\perp}^{2}, \quad(p<7), \\
F_{p+2} & =d H(r)^{-1} \wedge d x^{0} \wedge d x^{1} \wedge \ldots \wedge d x^{p}, \\
e^{\Phi} & =g_{s} H_{p}(r)^{\frac{3-p}{4}},
\end{aligned}
$$

with

$$
H_{p}(r)=1+\frac{\alpha}{r^{7-p}} \quad \text { and } \quad \alpha=(4 \pi)^{\frac{5-p}{2}} \Gamma\left(\frac{7-p}{2}\right)\left(\alpha^{\prime}\right)^{\frac{7-p}{2}} g_{s} N
$$

\section{Comments:}

- First, apart from the above solutions, there are more solutions to classical SUGRA. For instance, there are solutions to type II theories which are charged under the Kalb-Ramond B-field. Exmples are the fundamental F1 string and the NS5 brane. The geometry of fundamental F1 string, corresponding to $p=1$, is given as

$$
\begin{aligned}
d s^{2} & =H(r)^{-\frac{3}{4}} d x_{\|}^{2}+H(r)^{\frac{1}{4}} d x_{\perp}^{2}, \\
B_{(2)} & =\left(H(r)^{-1}-1\right) d x^{0} \wedge d x^{1}, \quad e^{\Phi}=g_{s} H(r)^{-1 / 2}
\end{aligned}
$$

with

$$
H(r)=1+\frac{\alpha}{r^{6}} \quad \text { and } \quad \alpha=32 \pi^{2}\left(\alpha^{\prime}\right)^{3} g_{s} N .
$$

In the string frame, one can rewrite the metric as $d s^{2}=H(r)^{-1} d x_{\|}^{2}+d x_{\perp}^{2}$ for which the curvature scales as $R \sim r^{-2}$. This shows that the curvature diverges at the core of the fundamental string. However $e^{\Phi}$ approaches a vanishing limit at the core. At an asymptotically far away regime from the fundamental string, $\Phi \rightarrow \Phi_{0}$, so that $g_{s} \rightarrow 0$. In this regime, the fundamental string is weakly coupled and perturbation theory make sense. The opposite is the case for the magnetic dual of the fundamental F1 string called the NS5 brane, charcterized by coupling $e^{\Phi}=g_{s}\left(1+\frac{\alpha}{r^{2}}\right)^{1 / 2}$, the metric $d s^{2}=\left(1+\frac{\alpha}{r^{2}}\right)^{-\frac{1}{4}} d x_{\|}^{2}+$ $\left(1+\frac{\alpha}{r^{2}}\right)^{\frac{3}{4}} d x_{\perp}^{2}$ and $B_{(6)}=\left(\left(1+\frac{\alpha}{r^{2}}\right)^{-1}-1\right) d x^{0} \wedge \ldots \wedge d x^{5}$. At the core of this object, $e^{\Phi}$ diverges, making NS5 brane a non-perturbative object and thus, a solitonic solution.

- D1 and D5 shares the same metric with the fundamental F1 string and NS5 brane respectively, but are related differently to the dilaton and thus, their couplings.

- Other brane solution can be straightfowardly constructed out from (89). One should note that the electric D3 brane turns out to be its own magnetic dual. This self dual object will play a very important role later in AdS/CFT correspondence, which is our ongoing topic of discussion. What is more is the important realization that for $p=3$, the dilaton is constant i.e. $e^{\Phi}=g_{s}$.

\begin{tabular}{||l||l||}
\hline Theory & Brane solutions \\
\hline Type IIA Superstring & D0, D2, D4, D6, D8 \\
\hline Type IIB Superstring & D(-1), D(1), D(3), D(5), D(7) \\
\hline M-theory & M2, M5 \\
\hline
\end{tabular}

Table 2. Elementary $p$-brane solutions. 


\subsection{The Near-Horizon Geometries}

Earlier in the lecture, we introduced the near-horizon geometry of the extremal RN black hole. The geometry of the near-horizon is $A d S_{2} \times S^{2}$ (cf. section 1.1.1). In general, any near-horizon geometry of the $p$-brane solution has the form $A d S_{p+2} \times X^{d-p-2}$. Now, we would like to know the corresponding near-horizon geometry of the $p$-brane configurations constructed in the last section.

Let us start by reminding ourselves that the $p$-brane solution we constructed has the property that it is asymptotically flat i.e. the solution asymptotically approaches the flat Minkowski space $\mathbb{R}_{1}^{d}$ far away from the brane. This can be further understood from the boundary condition we imposed in (74) and the fact that the solution features only an harmonic function $H(r)$ for which $\lim _{r \rightarrow \infty} H(r)=1$. Thus, the various $p$-brane solutions interpolate between the flat Minkowski space $\mathbb{R}_{1}^{d}$ and the near-horizon geometry $A d S_{p+2} \times X^{d-p-2}$. What is then the compact manifold $X^{d-p-2}$ admitted by the various $p$-brane solutions?

Let us look for the near-horizon geometry of the D3 brane in type IIB superstrin theory. The geometry of D3 brane, corresponding to $p=3$ in solution (89), is given as

$$
\begin{aligned}
d s^{2} & =H(r)^{-\frac{1}{2}} d x_{\|}^{2}+H(r)^{\frac{1}{2}} d x_{\perp}^{2}, \\
F_{5} & =(1+\star) \epsilon_{\mathbb{R}^{1,3}} \wedge H(r)^{-1}, \quad e^{\Phi}=g_{s},
\end{aligned}
$$

with

$$
H(r)=1+\frac{\alpha}{r^{4}} \quad \text { and } \quad \alpha=4 \pi\left(\alpha^{\prime}\right)^{2} g_{s} N .
$$

This solution corresponds to $N$ parallel D3 branes at sourced at $r=0$ and further enjoys $\mathrm{SO}(1,3) \times \mathrm{SO}(6)$ symmetry. We can write the metric more explicitly as

$$
d s^{2}=H(r)^{-\frac{1}{2}} d x_{(1+3)}^{2}+H(r)^{\frac{1}{2}}\left[d r^{2}+r^{2} d \Omega_{(5)}^{2}\right],
$$

where $\Omega_{(5)}^{2}$ is the metric of the $S^{5}$. By setting $\alpha=r_{3}^{4}$ and then demand the near horizon limit, one observes that

$$
\begin{aligned}
\lim _{r \rightarrow 0} H(r)^{-1 / 2} & =\lim _{r \rightarrow 0}\left(1+\frac{r_{3}^{4}}{r^{4}}\right)^{-1 / 2}=\left(\frac{r}{r_{3}}\right)^{2}, \\
\lim _{r \rightarrow 0} H(r)^{1 / 2} & =\lim _{r \rightarrow 0}\left(1+\frac{r_{3}^{4}}{r^{4}}\right)^{1 / 2}=\left(\frac{r}{r_{3}}\right)^{-2}, \\
\lim _{r \rightarrow 0} r^{2} H(r)^{-1 / 2} & =r_{3}^{2},
\end{aligned}
$$

so that the geometry in the near-horizon limit is given as

$$
d s^{2} \simeq\left(\frac{r}{r_{3}}\right)^{2} d x_{(1+3)}^{2}+\left(\frac{r_{3}}{r}\right)^{2} d r^{2}+r_{3}^{2} d \Omega_{(5)}^{2} .
$$

A change of variable $u=\frac{r_{3}^{2}}{r}$ and up to redefinition led to the near horizon metric

$$
d s^{2} \simeq r_{3}^{2} \frac{d x_{(1+3)}^{2}+d u^{2}}{u^{2}}+r_{3}^{2} d \Omega_{(5)}^{2} .
$$

Obviously, the first term describes an $A d S_{5}$ space with radius $r_{3}$, so that the near-horizon geometry of D3 brane has a topology $A d S_{5} \times S^{5}$. Thus, D3 brane interpolate between the flat 
Minkowski space $\mathbb{R}_{1}^{10}$ and the near-horizon geometry $A d S_{5} \times S^{5}$. This can be generalized to any $p$-brane solution in superstring or M-theory. This is a very important result. Indeed, it was conjectured by Maldacena [28] that the large $N$ limit of the $\mathcal{N}=4$ super Yang-Mills theory is dual to the type IIB superstring theory on $A d S_{5} \times S^{5}$. Understanding this idea is, in fact, our starting motivation for for this lecture.

The three $p$-brane solutions we constructed above have the metric of the form

$$
d s^{2}=H(r)^{-\gamma_{1}} d x_{\|(p+1)}^{2}+H(r)^{\frac{2}{\gamma_{2}}} d x_{\perp}^{2}
$$

with

$$
H(r)=1+\left(\frac{\alpha}{r}\right)^{\gamma_{2}}
$$

where $\gamma_{1}=1-\frac{2}{\gamma_{2}}$, with $\gamma_{2}=4,6,3$ for D3, M2 and M5 branes respectively. Following a similar evaluation, the near horizon geometry of supermembranes can be constructed. The summary of the near horizon geometries is contained in table 3 :

\begin{tabular}{||l||l||}
\hline Brane Solution & Near-horizon Topology \\
\hline D3-brane & $A d S_{5} \times S^{5}$ \\
\hline M2-brane & $A d S_{4} \times S^{7}$ \\
\hline M5-brane & $A d S_{7} \times S^{4}$ \\
\hline
\end{tabular}

Table 3. The near-horizon geometry of some $p$-brane solutions.

\section{Comment:}

One observe that the near-horizon geometries of these brane solutions can be brought to the form

$$
A d S_{p+2} \times S^{d-p-2} .
$$

However, we emphazise that the near-horizon geometry of the Dp-brane in the type II string theories are not generally of the form (101). In fact, D3 brane is only an exception. For $p \neq 3$, the near horizon geometry is only conformal to (101), with singular non-trivial conformal factor for $p<3$ and zero for $p>3$. Rather, geometries with topology

$$
A d S_{p+2} \times S^{D} \times \mathcal{M}_{d-p-2},
$$

for some space $\mathcal{M}$, arise as the near horizon solution of the Dp-brane. One can then arrive at $A d S_{p+2} \times S^{D}$ geometry by compactifying on $\mathcal{M}$.

Further account on the near horizon geometries can be found in $[29,30]$. The near horizon geometry of coincident KK-monopoles in 11D SUGRA is further discussed in [31].

\subsection{Special Holonomy Manifold in M-theory}

Let us make a return back to $d=11$ SUGRA which is the low energy limit of M-theory. The bosonic part of the action is given in (60) as

$$
2 \kappa_{11}^{2} S=\int d^{11} x \sqrt{-G}\left(R-\frac{1}{2} F_{4} \wedge \star F_{4}\right)-\frac{1}{6} \int C_{3} \wedge F_{4} \wedge F_{4}
$$


with the corresponding field equations

$$
\begin{aligned}
d \star F_{4} & =\frac{1}{2} F_{4} \wedge F_{4}, \\
R_{M} N-\frac{1}{2} G_{M N} R & =T_{M N}(C), \\
d G & =0 .
\end{aligned}
$$

which are invariant under SUSY transformations

$$
\begin{aligned}
& \delta_{\epsilon} G \sim \epsilon \Psi, \quad \delta_{\epsilon} C_{3} \sim \epsilon \Psi \\
& \delta_{\epsilon} \Psi \sim \nabla \epsilon+\epsilon \Psi \Psi .
\end{aligned}
$$

Earlier on, we have sought for the bosonic configurations that preserves some amount of SUSY i.e. solution with $\Psi=0$ that are invariant under (105) such that

$$
\delta_{\epsilon} \Psi_{M} \equiv \nabla_{M} \epsilon+\frac{1}{288}\left(\Gamma_{M}^{P Q R S} F_{P Q R S}-8 \Gamma^{P Q R} F_{M P Q R}\right) \epsilon=0 .
$$

In particular, we saw a class of two elementary supermembrane solutions of this theory, namely the electric M2-brane and its magnetic dual, M5-brane, which are BPS as they preserve 1/2SUSY. The appearance of the non-trivial solution of $C_{3}$ or equivalently $F_{4}$ in these solutions implies that these supermembranes are sourced by $C_{3}$ and thus have non-vanishing $F_{4}$ flux.

Nevertheless, for vanishing 4-form flux, there is a different class of supersymmetric configurations of $\mathrm{D}=11$ SUGRA. In this case, we seek for configurations satisfying

$$
R_{M N}=0, \quad \nabla_{M} \epsilon=0 .
$$

These are nothing but Ricci-flat manifolds that admit covariantly constant spinors $\epsilon$. Solution to (107) are classified by their holonomy group and they play important roles in M-theory.

Of important phenomenology interest in higher dimensional theory is to make contact with the physics in 4 or lower dimensions. In doing so, one compactify the theory on the manifolds in the extradimensions. We shall now go by the fact that the admittance of covariantly constant spinor gaurantees that an $d=11$ background manifold of the form $\mathbb{R}^{1,10-D} \times X_{D}$ solves $d=11$ SUGRA. Thus, the metric $G_{M N}$ take the structure of (72) i.e. as a product of Minkowski metric $\mathbb{R}^{1,10-D}$ and euclidean metric $g_{M N}(X)$ on $X_{D}$. As a reminder, this prescription of the $11 \mathrm{D}$ metric $G_{M N}$ would explicitly break the origninal $S O(1,10)$ to $S O(1,10-D) \times S O(D)$ (cf. (22)). In this regard, we split the the spinorial SUSY parameter as $\epsilon=\eta \otimes \theta$, where $\eta$ is the basis of the covariant constant spinor on the minkowski space and $\theta$ the corresponding one on $X$. In order to retain some amount of SUSY, it is thus sufficient to seek for those configurations admitting $\theta$ such that

$$
\nabla_{g(X)} \theta=0
$$

Manifolds admitting this conditions thus have $\mathrm{Hol}(g(X))$. On $X_{D}$, the holonomy group $\mathrm{Hol}(g(X))$ is a proper subgroup of the special orthogonal group $S O(D)$, and their choices are very limited to few classes according Berger [32]. The possible holonomy groups for simply connected Riemannian manifolds $X_{D}$ that admit covariantly constant spinor $\theta$ are listed in table 4 .

Thus, we see that for $D=7,8$, there are respectively $X_{D}=G_{2}$, Spin(7) manifolds such that the background manifold of the form $\mathbb{R}^{1,10-D} \times X_{D}$ solves $d=11$ SUGRA. Compactification of M-theory on $G_{2}$ manifold and $\operatorname{Spin}(7)$ manifold respectively yield $d=4$ and $d=3$ QFT. For $S U(n)$-Holonomy manifold in $D=2 n$, the relevant case for $d=11$ SUGRA are those 


\begin{tabular}{||l||l||l||l||}
\hline $\operatorname{dim}_{\mathbb{R}} X_{D}$ & Manifold $X_{D}$ & Metric $\mathbf{g}_{M N}(X)$ & $\mathbf{H o l}(\mathbf{g}(\mathbf{X}))$ \\
\hline$n$ & Orientable manifold $T_{n}$ & & $S O(n)$ \\
\hline $2 n$ & Kähler manifold & Kähler & $U(n)$ \\
\hline $2 n$ & Calabi-Yau $n$-fold $C Y_{n}$ & Ricci-flat, Kähler & $S U(n)$ \\
\hline $4 n$ & Quaternion-Kähler & Einstein & $S p(n) . S p(1)$ \\
\hline $4 n$ & Hyperkähler $H K_{n}$ & Ricci-flat, Kähler & $S p(n)$ \\
\hline 7 & $G_{2}$ manifold & Ricci-flat & $G_{2}$ \\
\hline 8 & Spin(7) manifold & Ricci-flat & Spin $(7)$ \\
\hline
\end{tabular}

Table 4. Classification of Berger's holonomy groups.

Calabi-Yau $n$-fold $\left(C Y_{n}\right)$ with $n=2,3,4,5$. In the case of $S p(n)$-Holonomy manifold in $D=4 n$, only $n=1,2$ Hyperkähler manifold $\left(H K_{n}\right)$ are relevant for $d=11$ SUGRA.

For these classical configurations, one then ask the question about the amount of SUSY they preserve. The answer to this has to do with the relationship between the holonomy of a manifold solution and the supersymmetric nature of it. Basically, holonomy is a geometric feature of a manifold that describes the amount of symmetry of the manifold. For our case, the amount of SUSY preserved by $X_{D}$ is related to its holonomy group. The larger the holonomy group of $X_{d}$, the smaller the fraction of SUSY it preserves. In [33], M-theory compactifications on manifolds of exceptional holonomy was reviewed, and a relationship between the holonomy group and SUSY was highlighted. This we summarize in table 5. More on this is contained in [34].

\begin{tabular}{||l||l||l||l||l||}
\hline Manifold $X_{D}$ & $T_{n}$ & $C Y_{3}$ & $X_{G_{2}}$ & $X_{\text {Spin }}(7)$ \\
\hline $\operatorname{dim}_{\mathbb{R}} X_{D}$ & $n$ & 6 & 7 & 8 \\
\hline$H \operatorname{Hol}\left(X_{D}\right)$ & $\mathbf{1}$ & $\mathrm{SU}(3)$ & $G_{2}$ & $\operatorname{Sppin}(7)$ \\
\hline $\operatorname{SUSY}(\nu)$ & 1 & $1 / 4$ & $1 / 8$ & $1 / 16$ \\
\hline
\end{tabular}

Table 5. The amount of SUSY preserved by some holonomy manifolds. As the holonomy get larger, the fraction of SUSY preserved gets smaller.

\section{Acknowledgements}

I thank Johannes Walcher, Richard Eager, and Ingmar Saberi for discussions.

\section{References}

[1] Karl Schwarzschild, Über das Gravitationsfeld eines Massenpunktes nach der Einsteinschen Theorie, Sitzungsberichte der Königlich Preußischen Akademie der Wissenschaften 7 (1916) 189-196.

[2] H. Reissner, Über die Eigengravitation des elektrischen Feldes nach der Einsteinschen Theorie, Annalen der Physik 50 (1916) 106-120; G. Nordström, On the Energy of the Gravitational Field in Einstein's Theory, Koninklijke Nederlandsche Akademie van Wetenschappen Proceedings 20 (2) (1918) 1238-1245.

[3] Roy P. Kerr, Gravitational Field of a Spinning Mass as an Example of Algebraically Special Metrics, Phys. Rev. Lett. 11 (1963) 237-238.

[4] E. T. Newman and A. I. Janis, Note on the Kerr Spinning-Particle Metric, Journal of Mathematical Physics. 6 (6) (1965) 915-917.

[5] Friedrich Kottler, Über die physikalischen Grundlagen der Einsteinschen Gravitationstheorie, Annalen der Physik, vol. 361, Issue 14 (1918) 401-462 
[6] Carlo Rovelli, Notes for a brief history of quantum gravity, arXiv:gr-qc/0006061; A. Ashtekar, M. Reuter, C. Rovelli, From General Relativity to Quantum Gravity, In "General Relativity and Gravitation: A Centennial Perspective", arXiv:1408.4336; Lee Smolin, What are we missing in our search for quantum gravity?, arXiv:1705.09208

[7] Juan Martin Maldacena, The Large N limit of superconformal field theories and supergravity, Adv. Theor. Math.Phys. 2 (1998) 231-252.

[8] O. Aharony, S.S. Gubser, J. Maldacena, H. Ooguri, Y. Oz, Large N Field Theories, String Theory and Gravity, Phys. Rept. 323 (2000) 183-386.

[9] F. R. Tangherlini, Schwarzschild field in $n$ dimensions and the dimensionality of space problem, Nuovo Cim (1963) 27: 636.

[10] Hideo Kodama, Akihiro Ishibashi, A Master equation for gravitational perturbations of maximally symmetric black holes in higher dimensions, Prog. Theor. Phys. 110 (2003) 701-722.

[11] I. Antoniadis, The Physics of Extra Dimensions, Lect. Notes Phys. 720 (2007) 293-321.

[12] The many lives of supergravity, CERN Courier, January/February 2017, Volume 57, Number 1, $41-44$.

[13] Daniel Z. Freedman, Antoine Van Proeyen, Supergravity, Cambridge University Press (2012) ISBN: 9781139026833.

[14] Yoshiaki Tanii, Introduction to Supergravity, Springer Japan (2014) ISBN: 4431548270.

[15] John Terning, Modern Supersymmetry: Dynamics and Duality, OUP (2005) ISBN: 9780198567639.

[16] Matteo Bertolini, Lectures on Supersymmetry.

[17] J.A. Strathdee, Extended Poincare Supersymmetry, Int.J.Mod.Phys. A2 (1987) 273.

[18] Gary T. Horowitz, Andrew Strominger, Black strings and P-branes, Nucl. Phys. B360 (1991) 197-209.

[19] M.J. Duff, R.R. Khuri, J.X. Lu, String solitons, Phys. Rept. 259 (1995) 213-326.

[20] K.S. Stelle, Lectures on supergravity p-branes, C96-06-10.2, p.287-339 .

[21] E.S. Fradkin and A.A. Tseytlin, Effective Field Theory from Quantized Strings, Phys. Lett. B 158,316 (1985).

[22] E.S. Fradkin and A.A. Tseytlin, Quantum string theory effective action, Nucl. Phys. B261 (1985) 1-27.

[23] C.M. Hull, Exact pp Wave Solutions of Eleven-dimensional Supergravity, Phys. Lett. B139 (1984) 39.

[24] David J. Gross, Malcolm J. Perry, Magnetic Monopoles in Kaluza-Klein Theories, Nucl. Phys. B226 (1983) $29-48$.

[25] R.D. Sorkin, Kaluza-Klein Monopole, Phys. Rev. Lett. 51 (1983) 87-90.

[26] M.J. Duff, K.S. Stelle, Multimembrane solutions of D = 11 supergravity, Phys. Lett. B253 (1991) $113-118$.

[27] R. Güven, Black p-brane solutions of D = 11 supergravity theory, Phys.Lett. B276 (1992) 49-55.

[28] Juan Martin Maldacena, The Large $N$ limit of superconformal field theories and supergravity, Int. J.Theor. Phys. 38 (1999) 1113-1133.

[29] Jose M. Figueroa-O'Farrill, Near horizon geometries of supersymmetric branes, arXiv:hep-th/9807149.

[30] Bobby Samir Acharya, J.M. Figueroa-O'Farrill, C.M. Hull, Bill J. Spence, Branes at conical singularities and holography, Adv.Theor.Math.Phys. 2 (1999) 1249-1286.

[31] Nissan Itzhaki, Juan M. Maldacena, Jacob Sonnenschein, Shimon Yankielowicz, Supergravity and The Large $N$ Limit of Theories With Sixteen Supercharges, Phys. Rev. D 58, 046004 (1998).

[32] Marcel Berger, Sur les groupes d'holonomie homogènes de variétés à connexion affine et des variétés riemanniennes, Bulletin de la Société Mathématique de France 83 (1955): 279-330.

[33] Bobby S. Acharya, Sergei Gukov, $M$ theory and singularities of exceptional holonomy manifolds, Phys.Rept. 392 (2004) 121-189.

[34] Michael Douglas et. al., Strings and Geometry, Proceedings of the Clay Mathematics Institute 2002 Summer School. 